\title{
Modified pillow splint
}

Sir,

Injuries of the leg/foot are commonly seen in a busy reconstructive surgery unit. These are usually due to road traffic accidents. Many such injuries need flap cover. Difficult composite defects are increasingly being managed with microvascular flaps. An important factor to ensure consistent success in the postoperative period in a microvascular flap is proper positioning of the leg/foot.

Proper positioning is needed to:

1. Provide adequate elevation to counter edema and aid in venous return

2. Prevent direct pressure over the flapped area

3. Provide a stable support for the operated limb to prevent accidental sliding and falling of the leg

4. To meet these ends, we have tried various splints in our unit. Elevation over a pillow (two pillows in some cases) is a simple method to position an operated leg. However, it is not a very stable splint because patients have had their legs slipping down the pillow especially when they have been asleep. A Bohler Braun splint ${ }^{[1]}$ has also been useful, but again is not very stable. Use of Thomas Splint ${ }^{[2]}$ has been described. However, it does not allow the foot to be free in cases of circumferential coverage of foot defects with free flaps.

In search of better stability, we initially taped rolled bed sheets over the pillow adjacent to the leg. The tapes often loosened resulting in the rolled bed sheets

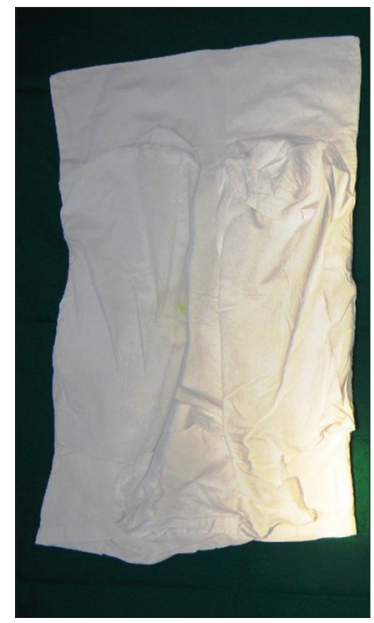

Figure 1a: The modified pillow cover 


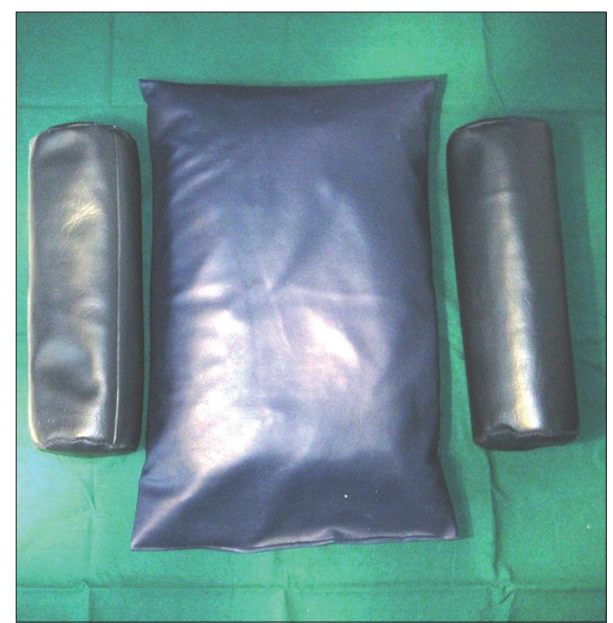

Figure 1b: The modified cylindrical pillows with regular pillow

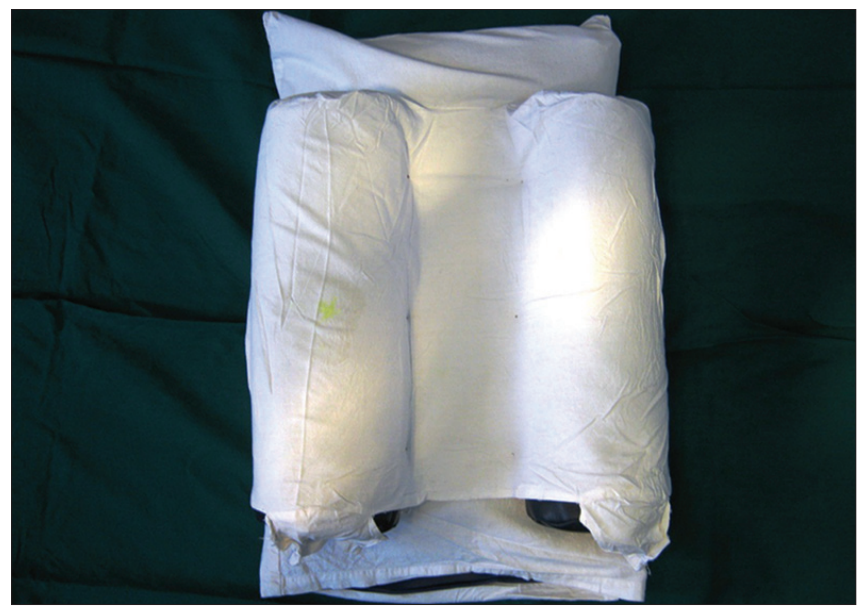

Figure 2: The assembled modified pillow splint

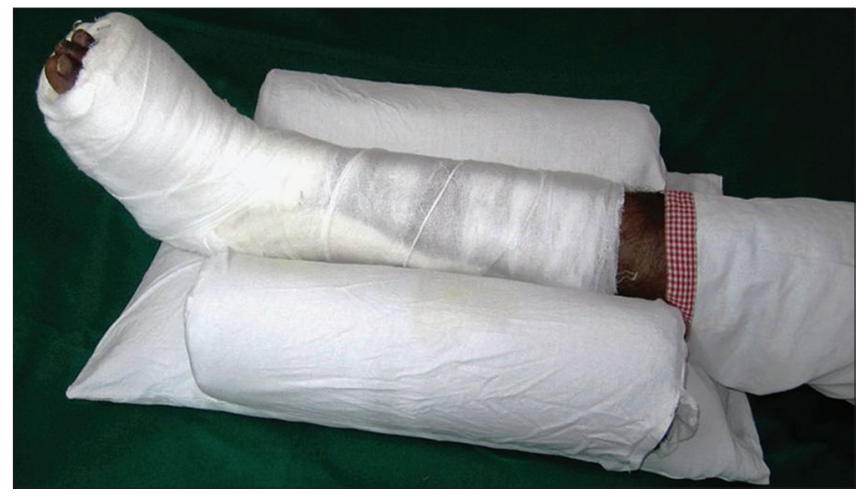

Figure 3: Use of the splint in a patient

dislodging from the pillow. The modified pillow has evolved from the same principle. The idea was to stitch two linen covers over a standard pillow cover which could hold two cylindrical-shaped smaller pillows. We described what we needed to the linen and maintenance departments in our hospital. The modified pillow cover was made with regular linen used for pillow covers and the pillows were made from foam within and rexine-like material outside. After a few trial and errors we were able to put in place the modified pillow splint. We have been using this splint for about 2 years now and find it useful in nursing legs covered with microvascular flaps.

The modified pillow splint consists of a modified pillow cover (to enable it to hold two additional cylindrical pillows) and two cylindrical pillows in addition to the regular pillow [Figures $1 \mathrm{a}$ and $1 \mathrm{~b}$ ]. The individual components can be washed separately and reassembled for use [Figures 2 and 3]. It fulfils the requirements for a good leg/foot splint. We have found it particularly useful to nurse circumferential degloving injuries of the foot which are covered with a microvascular flap. In these cases, the modified pillow splint can be positioned in such a way that the distal aspect of the splint is at the level of ankle and the foot is free without pressure on any surface of the foot.

A drawback of this splint is that it cannot be used in leg cases with an external fixator.

\section{Ellur Sunderraj}

Department of Plastic Surgery, ${ }^{1}$ Nursing Staff Incharge of Plastic Surgery Ward, St John's Medical College and Hospital, Bangalore, Karnataka, India

\section{Address for correspondence: Dr. Sunderraj Ellur, Department of Plastic Surgery, St John's Medical College Hospital, Bangalore - 34, Karnataka, India. E-mail: sundernetra@yahoo.co.in}

\section{REFERENCES}

1. Ninan S, Manigandan C, Gupta AK. Postoperative Care of Flaps Using the Bohler Braun Frame: An Innovation. Plast Reconstr Surg 2005;115:676-7.

2. Bhaskara KG, Kale SM. Use of Thomas splint in salvaging free flaps of the lower limb in violent postoperative patients. Indian J Plast Surg 2009;42:271-2.

\begin{tabular}{|l|l|}
\hline \multicolumn{3}{|c|}{ Access this article online } \\
\hline Quick Response Code: & Website: \\
\hline & www.ijps.org \\
\cline { 2 - 2 } & DoI: \\
\hline
\end{tabular}

